\title{
Soft tissue myxoma of the gingiva: Report of a case and review of the literature of soft tissue myxoma in the oral region
}

\author{
Tetsuo Shimoyama, Norio Horie, Takao Kato, Takahiro Tojo, \\ Daisuke Nasu, Takahiro Kaneko and Fumio Ide \\ Department of Oral Surgery, Saitama Medical Center \\ Saitama Medical School, Saitama 350-8550 \\ (Received 12 November 1999 and accepted 10 April 2000)
}

\begin{abstract}
Soft tissue myxoma of the oral cavity is extremely rare. We present a case of soft tissue myxoma arising from a mandibular anterior gingiva in a 51-yearold male patient. Histological examination showed islands of odontogenic epithelium scattered in the mucinous stroma. This lesion was supposed to have a odontogenic origin. The clinical differences between soft tissue myxoma with bone destruction and those without bone destruction are also discussed by a review of the literature. (J. Oral Sci. 42, 107-109, 2000)
\end{abstract}

Key words: soft tissue myxoma; gingiva; odontogenic tumor.

\section{Introduction}

Odontogenic myxoma is a rare benign tumor, which is locally invasive and has a high recurrence rate (1). This tumor commonly occurs in the body of the maxilla or mandible and is supposed to be of odontogenic mesenchymal origin (1). However, on rare occasions, this lesion may arise from soft tissue (2). We present an unusual case of odontogenic myxoma arising from a mandibular anterior gingiva.

\section{Case report}

A 51-year-old male patient consulted the oral surgery clinic of Saitama Medical Center to evaluate the gingival mass of the mandibular anterior region. About one year previously, the patient had noticed a mass the size of a rice

Correspondence to Dr. Norio Horie, Department of Oral Surgery, Saitama Medical Center, Saitama Medical School, 1981 Kamoda, Kawagoe, Saitama 350-8550, Japan grain on the labial gingiva. The mass had gradually enlarged then suddenly enlarged further, and the patient felt discomfort during mastication. His previous medical history was unremarkable.

On physical examination, a painless, pedunculated, granulomatous mass, measuring $30 \times 28 \mathrm{~mm}$ extruded from the labial gingiva in the mandibular anterior region (Fig. 1). The surface of the mass was ulcerative and showed traces of the upper tooth. The left lateral incisor exhibited severe mobility. Regional lymph nodes were not palpable. On radiographic examination, the left lateral incisor was tilted lingually. The alveolar bone between the lateral incisor and canine was moderately absorbed. The alveolar crest was not flat. The lamina dura and periodontal space of both teeth were partially obliterated. There was no sign of root resorption. The body of the mandible was intact, but the tumor perforated the labial bone cortex and fine bone trabeculation was found in the mass (Fig. 2). The

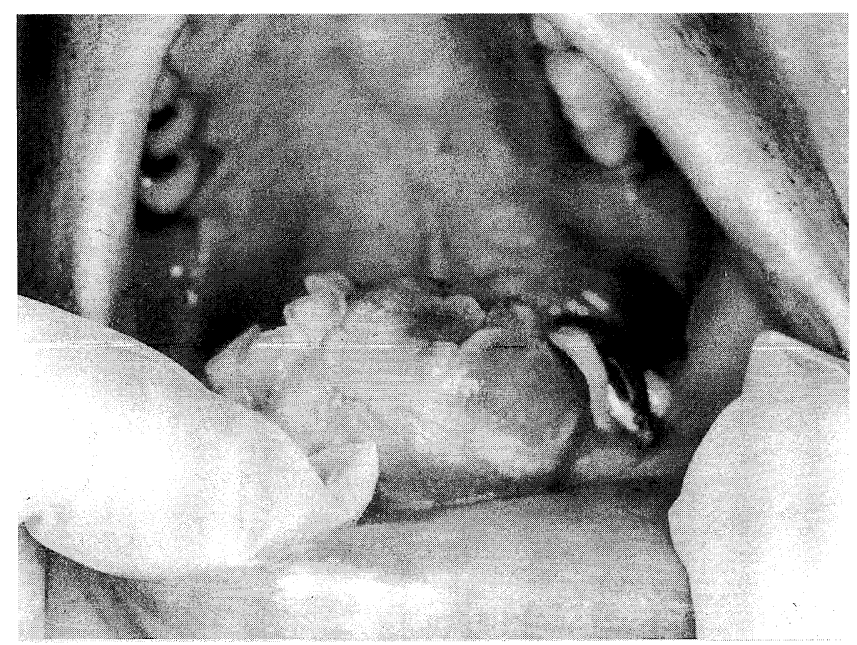

Fig. 1 Clinical appearance of the tumor. 


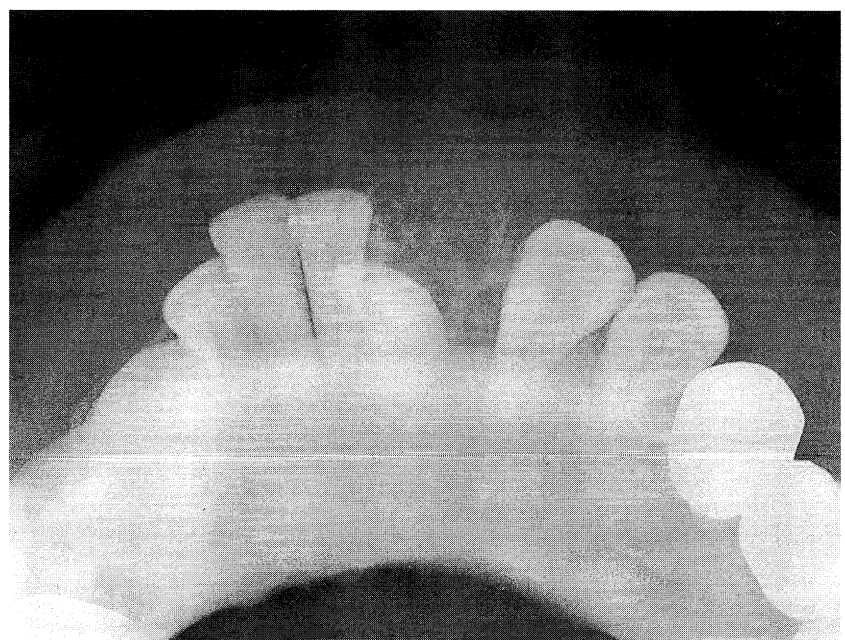

Fig. 2 Occlusal radiograph showing bony trabecular formation in the mass.

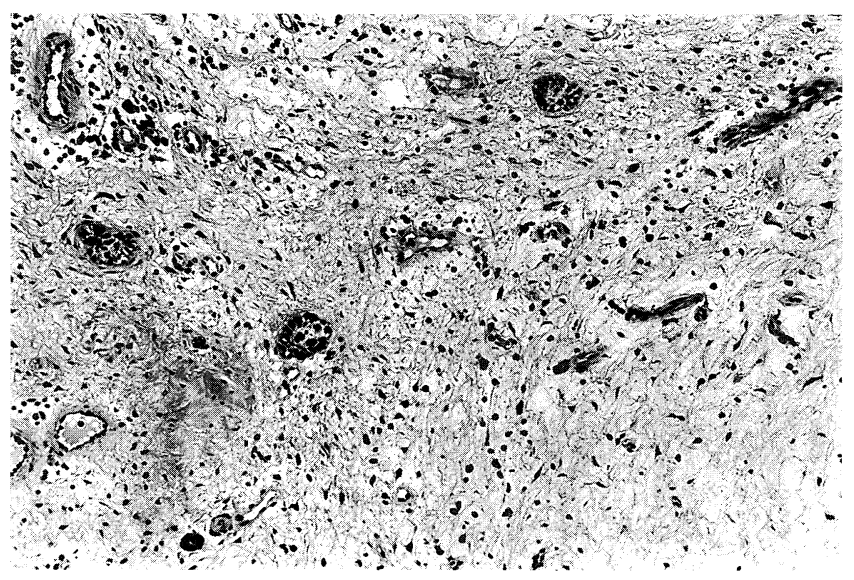

Fig. 3 Spindle and stellate cells were suspended on a myxomatous background. Islands of odontogenic epithelium were seen (HE; original magnification $\times 100)$.

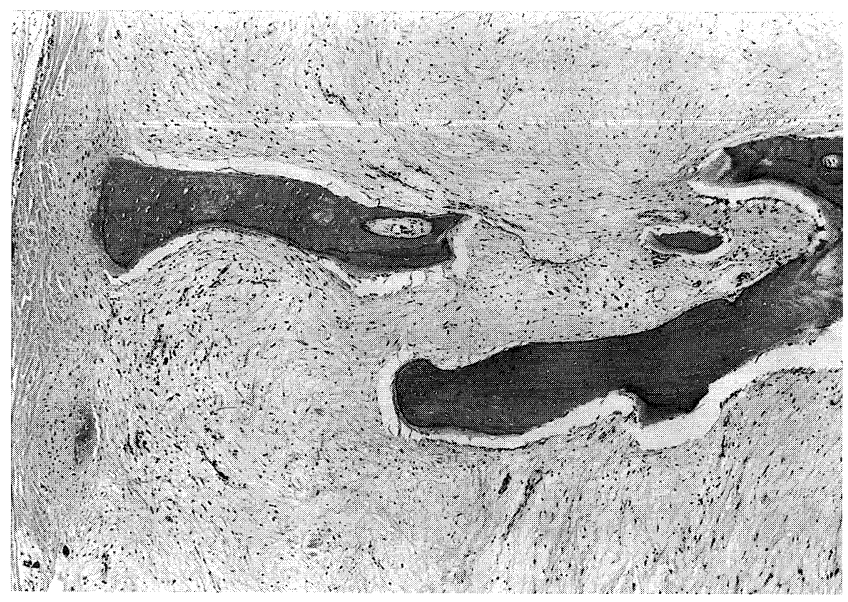

Fig. 4 Remnants of trabecular bone were seen dispersedly in the stroma $(\mathrm{HE}$; original magnification $\times 40$ ). clinical diagnosis was peripheral ossifying fibroma.

Under local anesthesia, the mass was resected with a margin of normal healthy tissue and the left lateral incisor and canine were removed with the tumor. Intraoperatively, the alveolar crest was coarse and adequate curettage was performed. Recovery was uneventful and there has been no recurrence during two years follow-up.

The resected mass measured $42 \times 25 \times 10 \mathrm{~mm}$, and the cut surface had a mucinous appearance. Microscopic examination revealed an unencapsulated myxomatous tumor with ill-defined margins. The tumor was composed of abundant mucinous stroma, sparse stellate and spindle cells with hyperchromatic nuclei and distinct cytoplasm, and a meshwork of fine collagen fibers (Fig. 3). In addition, foci of odontogenic epithlium were scattered in the lesion. There was no evidence of cellular pleomorphism or hypercellularity. Remnants of residual trabeculae of alveolar bone were seen dispersedly in the stroma (Fig. 4). The proliferating spindle and stellate cells were positive for vimentin and partially positive for $\alpha$-smooth muscle actin using avidin-biotin peroxidase complex (ABC, Vector Lab). However, immunostaining for S-100 protein and keratin was negative in these cells. Rests and islands of odontogenic epithelium showed positive reaction for keratin.

\section{Discussion}

The odontogenic myxomas of the jaw are divided into two types, central myxomas located intraosseously and peripheral (extraosseous or soft tissue) myxomas occurring in soft tissue overlying tooth-bearing areas of the mandible and the maxilla (1). Schmidseder et al. (3) classified all the myxomas arising from the alveolar process, regardless of whether there was bone destruction, as being the peripheral type. Our case involved a soft tissue myxoma with unique bony trabecular formation in the mass and was supposed to correspond to Schmidseders' peripheral type.

In this article, we divided the cases of soft tissue myxoma including our case into two groups: cases showing bone destruction, and those without bone destruction, and compared the clinical findings of these two groups. Available information is presented from nine cases with bone destruction and five cases without bone destruction (2-8). Patient ages in cases having bone destruction ranged from 15 years to 97 years. Four cases occurred in the sixth decade. Patient ages in cases without bone destruction were distributed from 27 years to 45 years with three cases appearing in the third decade. The male to female ratio among cases showing bone destruction was $6: 3$ and all cases without bone destruction were from female patients. Although the total number of the cases was small, 
the cases showing bone destruction tended to occur in older people with a male predilection compared to cases without bone destruction. There was no difference in clinical duration between these two groups. Though myxoma tends to grow slowly, the cases showing bone destruction enlarged rapidly within a short period in five of nine cases (3,5-7).

Generally, jawbone myxoma has a high recurrence rate (3). There has not been any recurrence reported among cases without bone destruction. Therefore, surgical excision with adequate margins may be required depending on the size and location of the tumor, especially in soft tissue myxomas with bone destruction.

It remains obscure whether the origin of myxoma of the jawbones is definitely odontogenic. However, histologically the presence of odontogenic epithelium suggests that this tumor might derive from an odontogenic origin (1). Only one case of soft tissue myxoma of the gingiva showed odontogenic epithelium (5). Our case also showed odontogenic epithelial islands scattered in the tumor and is supposed to be truly odontogenic.

Immunohistochemically, tumor cells of odontogenic myxoma stain with vimentin and $\alpha$-smooth muscle actin (9-11). The positive staining of $\alpha$-smooth muscle actin has suggested that tumor cells show not only fibroblastic but also myofibroblastic differentiation (9). Our findings were similar to those findings reported in other cases (9-11).

\section{References}

1. Gundlach, K.K.H. and Schulz, A. (1977) Odontogenic myxoma-clinical concept and morphological studies. J. Oral Pathol. 6, 343-358

2. Elzay, R.P. and Dutz, W. (1978) Myxoma of the paraoral-oral soft tissues. Oral Surg. Oral Med. Oral Pathol. 45, 246-254

3. Schmidseder, R., Groddeck, A. and Scheunemann, H. (1978) Diagnostic and therapeutic problems of myxomas (myxofibromas) of the jaws. J. Maxillofac. Surg. 6, 281-286

4. Campos, G.M., Grandini, S.A. and Lopes, R.A. (1980) Myxoma of oral soft tissue. Tumori 66, 655659

5. Nakano, Y., Yamamoto, H., Shiozaki, H., Ishii, T., Nakamura, T., Kaneko, K. and Otake, S. (1985) Peripheral myxoma of the right maxilla: a case and review of the Japanese literature. J. Nihon Univ. Sch. Dent. 27, 119-125

6. Siar, C.H., Ng, K.H., Devadas, V. and Patricia, M. (1986) Oral soft tissue myxoma. Report of case. J. Oral Med. 41, 256-258

7. Hori, S., Kurachi, Y., Yagami, K., Kanemoto, K., Goto, H., Nagumo, M. and Yamana, H. (1987) Two cases of soft myxoma of the gingival region. Nihon Koku Geka Gakkai Zasshi 33, 1436-1441 (in Japanese)

8. Günhan, Ö., Arpak, N., Celasun, B. and Can, C. (1991) Odontogenic myxoma. Report of a periodontally-located case. J. Periodontol. 62, 387389

9. Moshiri, S., Oda, D., Worthington, P. and Myall, R. (1992) Odontogenic myxoma: histochemical and ultrastructural study. J. Oral Pathol. Med. 21, 401403

10. Lombardi, T., Lock, C., Samson, J. and Odell, E. W. (1995) S100, $\alpha$ - smooth muscle actin and cytokeratin 19 immunohistochemistry in odontogenic and soft tissue myxomas. J. Clin. Pathol. 48, 759-762

11. Lo Muzio, L., Nocini, P., Favia, G., Procaccini, M. and Mignogna, M.D. (1996) Odontogenic myxoma of the jaws: a clinical, radiologic, immunohistochemical, and ultrastructural study. Oral Surg. Oral Med. Oral Pathol. Oral Radiol. Endod. 82, 426-433 